\title{
Peut-on encore parler de sécheresse au Sahara ?
}

\author{
C. Thirriot \\ Institut de mécanique des fluides \\ ENSEEIHT, Toulouse
}

\author{
A. Matari \\ Institut hydrométéorologique \\ de la formation et de la recherche, Oran
}

\section{Introduction}

Le jardinier parisien habitué aux $750 \mathrm{~mm}$ de pluie annuelle moyenne du parc Montsouris décrétera qu'au Sahara c'est toujours la sécheresse. Mais pour la sécheresse c'est comme pour la pauvreté, le seuil varie avec l'opulence de l'environnement.

Les années de disette en France comme 1976, 1986, 1987 seraient encore un miracle au sud de l'Atlas. Alors en climat aride comment discerner les cas de sécheresse ?

Comme en économie, on peut choisir les deux points de vue de la ressource et de l'emploi. La ressource sera jaugée à l'aune statistique mais avec le handicap d'une information clairsemée dans le temps et dans l'espace. L'emploi est manifesté par la quête patiente et fataliste de l'herbe rare et râpeuse par le nomade qui conduit son troupeau le long des parcours sahariens.

\section{Qu'est donc la sécheresse ?}

\subsection{Sécheresse et aridité}

Le petit Larousse n'est d'aucun recours puisqu'il annonce comme définitions de Sécheresse : 1) état de ce qui est sec, 2) absence de pluie.
La première définition est la constatation d'un état, la deuxième la constatation d'un événement (ou de son complémentaire). En général cet événement est la cause de cet état-là. Comparé aux zones tempérées, on serait tenté de décréter l'état de sécheresse sur le Sahara. Mais ce qui nous intéresse ici, c'est l'événement de sécheresse sur une longue période. Pour l'état moyen que l'on considère le plus souvent sous le nom de climat, nous dirons que la caractéristique est l'aridité qui sera cette fois définie par comparaison avec les climats des autres zones telle la zone diversement tempérée de notre pays. Par contre, l'événement de sécheresse sera un aléa dans le climat d'une zone donnée qui durera un temps plus ou moins long avec une rigueur plus ou moins grande. Pour décréter qu'il y a sécheresse, il faudra donc constater des circonstances exceptionnelles ou tout au moins peu fréquentes par rapport à la situation climatique moyenne. En retenant la définition fondée sur le manque de pluie, on voit donc qu'on ne peut parler de sécheresse que s'il pleut, même peu. Et fort heureusement il pleut sur le Sahara, pas beaucoup certes, de manière erratique dans le temps et dans l'espace. Ces pluies capricieuses mais sources de vie ont fasciné des générations d'observateurs ou de météorologues tels par exemple Jean DUBIEF qui a consacré toute sa vie professionnelle à l'étude du climat saharien et qui encore aujourd'hui à plus de quatrevingt-cinq ans a été pour nous un initiateur aussi bien par

\section{Can one still talk of drought in the Sahara}

Even in arid zones, the temporal variability of rare and minimum, but sometimes very violent, rainfall, has important consequences on the mainly pastoral economic life.

The authors study the characteristics of dry and rainy sequences along the Alger-Kano axis showing up the specific Hoggar effect.

By taking into account the various definitions of drought, they examine their significance for the climate of the Sahara desert. 
Tableau 1. Liste des stations professionnelles.

\begin{tabular}{|l|c|c|c|c|c|}
\hline \multicolumn{1}{|c|}{ STATIONS } & LATITUDE & LONGITUDE & ALTITUDE & PERIODE & REMARQUES \\
\hline $\begin{array}{l}\text { DAR EL BEIDA } \\
\text { (Aérodrome) SP } \\
\text { ALGER }\end{array}$ & $\begin{array}{l}36^{\circ} 70 \mathrm{~N} \\
36^{\circ} 72 \mathrm{~N}\end{array}$ & $\begin{array}{l}03^{\circ} 23 \mathrm{E} \\
03^{\circ} 25 \mathrm{E}\end{array}$ & $\begin{array}{l}22 \mathrm{~m} \\
24 \mathrm{~m}\end{array}$ & $\begin{array}{l}1935-1955 \\
1956-1984\end{array}$ & REGULIERE \\
\hline $\begin{array}{l}\text { EL GOLEA } \\
\text { (Aérodrome) SP }\end{array}$ & $30^{\circ} 57 \mathrm{~N}$ & $02^{\circ} 87 \mathrm{E}$ & $397 \mathrm{~m}$ & $1930-1984$ & REGULIERE \\
\hline $\begin{array}{l}\text { DJANET SP } \\
\text { (VILLE) }\end{array}$ & $24^{\circ} 58 \mathrm{~N}$ & $09^{\circ} 47 \mathrm{E}$ & $1054 \mathrm{~m}$ & $1930-1938$ \\
\hline $\begin{array}{l}\text { IIELES RCP } \\
\text { (APC) }\end{array}$ & $23^{\circ} 82 \mathrm{~N}$ & $05^{\circ} 93 \mathrm{E}$ & $1400 \mathrm{~m}$ & $1970-1975$ & LACUNAIRE \\
\hline $\begin{array}{l}\text { IRHAFOC PP } \\
\text { (APC) }\end{array}$ & $23^{\circ} 67 \mathrm{~N}$ & $05^{\circ} 75 \mathrm{E}$ & $1470 \mathrm{~m}$ & $1970-1976$ & IRREGULIER \\
\hline $\begin{array}{l}\text { TAZROUK PCP } \\
\text { (APC) }\end{array}$ & $23^{\circ} 42 \mathrm{~N}$ & $06^{\circ} 27 \mathrm{E}$ & $1800 \mathrm{~m}$ & $1970-1978$ & IRREGULIER \\
\hline $\begin{array}{l}\text { ASSEKREM PCP } \\
\text { (ERMITAGE) }\end{array}$ & $23^{\circ} 27 \mathrm{~N}$ & $05^{\circ} 63 \mathrm{E}$ & $2585 \mathrm{~m}$ & $1955-1984$ & PEU LACUN. \\
\hline $\begin{array}{l}\text { TAMANRASSET SP } \\
\text { (OBSERVATOIRE) } \\
\text { TAMANRASSET }\end{array}$ & $22^{\circ} 79 \mathrm{~N}$ & $05^{\circ} 53 \mathrm{E}$ & $1377 \mathrm{~m}$ & $1939-1946$ & REGULIERE \\
\hline \begin{tabular}{l} 
KANO SP \\
\hline
\end{tabular} & $12^{\circ} 03 \mathrm{~N}$ & $08^{\circ} 32 \mathrm{E}$ & $476 \mathrm{~m}$ & $1955-1974$ & REGULIERE \\
\hline
\end{tabular}

SP : STATION PROFESSIONNELLE PCP : POSTE CLIMATOLOGIQUE PRINCIPAL PP : POSTE PLUVIOMETRIQUE sa conversation, son courrier que ses publications scientifiques dont en particulier les deux magnifiques tomes de son ouvrage "Le climat du Sahara" (1959). Bien souvent au cours de cet exposé nous nous référerons explicitement ou implicitement à l'œuvre de ce physicien enthousiaste et encore infatigable lorsqu'il s'agit de pluviométrie sur le Hoggar.

Commençons par régler la question du climat et de l'aridité. Les météorologues maghrébins parlent de climat aride lorsque le volume de pluie annuel n'atteint pas $300 \mathrm{~mm}$. Avec E. de MARTONNE on peut introduire l'indice d'aridité (LAMBERT, 1983),

$$
i_{a}=P /(T+10)
$$

$P$ est la pluviométrie annuelle

$T$ est la température Celsius moyenne.

\subsection{Sécheresse des géographes}

D'après le professeur LAMBERT, professeur de géographie à l'Université Toulouse Mirail, on peut distinguer quatre types de sécheresse :

1. la sécheresse atmosphérique lorsque l'atmosphère prélève plus d'eau qu'elle n'en apporte pendant une certaine durée. Le volume de pluie $P$ est inférieur au volume $E$ évaporé ou évapotranspiré ;

2. la sécheresse pédologique lorsque le sol se dessèche jusqu'à un certain seuil - par exemple le seuil de flétrissement correspondant à un $\mathrm{pF}$ de 4,2 soit une succion approximative de 15 bars ;

3. la sécheresse potamologique lorsque, à l'étiage, l'écoulement dans les cours d'eau s'amenuise et va jusqu'à disparaître ;

4. la sécheresse géologique lorsque les puits se tarissent.

\subsection{Sécheresse pastorale}

Au Sahara, en dehors des oasis, il n'est pas question de culture. La vie agricole est celle des troupeaux qui vont suivre le parcours des pâturages.

En Algérie seule, les pâturages jalonnent 40 millions d'hectares dont douze dans la steppe et faisaient vivre il y a encore quelques années 250000 familles. La ressource dans la steppe est estimée à 1,6 milliard d'unités de fourrage soit 120 à 150 unités de fourrage par hectare fortement dépendantes des aléas du climat continental (CHELLIG, 1974).

Les spécialistes d'économie saharienne parlent de cycle climatique d'environ cinq ans: une année de fourrage abondant, trois années moyennes et une année de disette au cours de laquelle le bétail est décimé : la totalité des agneaux est sacrifiée pour sauver les mères et malgré cela $50 \%$ des bêtes adultes meurent.

La pluie va régler la concentration et la vitesse de parcours des troupeaux, variable dans le temps et dans l'espace suivant les aléas. 


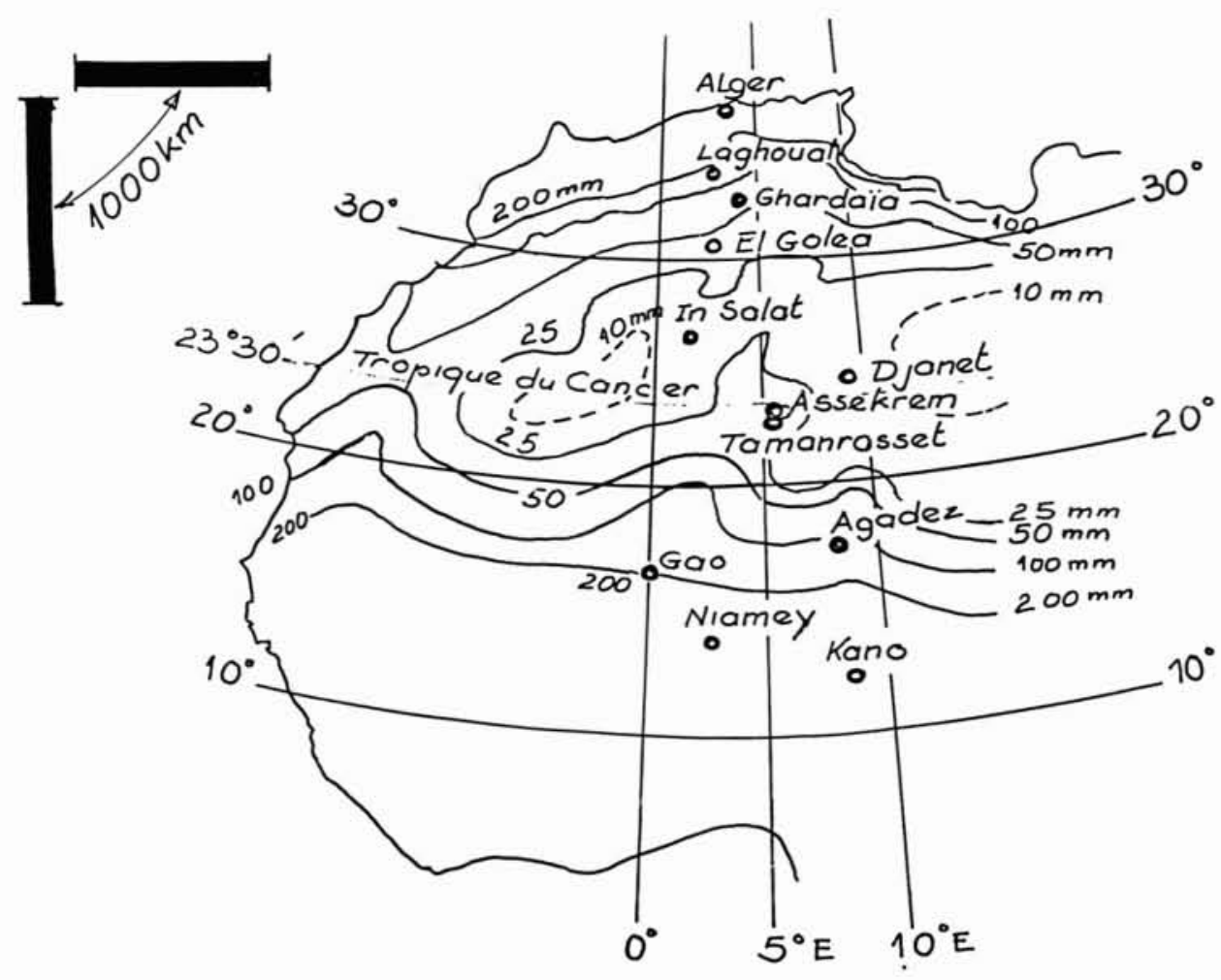

1. Carte de situation avec isohyètes annuelles.

A Tamanrasset la période de retour du pâturage abondant est de 15 ans alors que le pâturage moyen est observé tous les deux ans. A Djanet et El Goléa le pâturage moyen est beaucoup plus rare puisque grosso modo il n'est assuré que tous les cinq ans.

\subsection{Sécheresse statistique}

Cycle, fréquence, période de retour, ces mots qui interviennent déjà à propos des successions d'années de brebis maigres et d'années de brebis grasses font glisser du parler pastoral au langage statistique, refuge de ceux qui ont besoin de la sécurité mathématique face aux aléas cruels voire mortels de la nature.

\section{Que sait-on sur la pluviométrie saharienne? (Les sources d'information)}

Le territoire est immense, la pluie rare, capricieuse, souvent localisée, les reliefs provoquent de fortes particularités. Autant de circonstances qui rendent malaisée l'appréhension de la pluviométrie saharienne.

Cependant, l'histoire est déjà longue des observations pluviométriques bénévoles ou organisées. H.P. EYDOUX (1943) cite un relevé de $75 \mathrm{~mm}$ en deux jours à Aïn Sefra en 1904. Du début du siècle aussi datent les observations de Charles de Foucauld dans son refuge d'ermite au Hoggar. La mise en place d'un réseau de postes pluvio- métriques est à peine plus tardive puisque J. DUBIEF dans son magnifique ouvrage parle des séries de mesures concomitantes à partir de 1926. Il signale l'implantation de 26 pluviomètres totalisateurs blindés sur la partie du Sahara sous observation française installés par la D.H.E.R. et l'O.C.R.S, ainsi que quelques dizaines placés temporairement par l'O.R.S.T.O.M. en Ennedi, au Tibesti et en Aïr. Lui-même indique qu'il a réparti plus d'une centaine de pluviomètres plus rustiques dans le centre du Hoggar. Dans ses observations il y a là une mine d'informations peu exploitées et qui serait un bel argument pour la géostatique.

Actuellement l'Office national de la météorologie algérien dispose dans son réseau de stations de type professionnel (SP où sont relevés tous les paramètres climatiques habituels) et des stations auxiliaires suivies par des observateurs bénévoles. Les stations principales relèvent les quantités d'eau recueillie toutes les trois heures sauf par forte chaleur où la consigne est de noter la hauteur immédiatement après la fin de la pluie. Sont notés aussi les temps de début et fin de précipitation (MATARI, 1988).

Les stations dont les résultats sont surtout utilisés dans ce rapport sont présentés dans le tableau 1 et la planche 1 . 


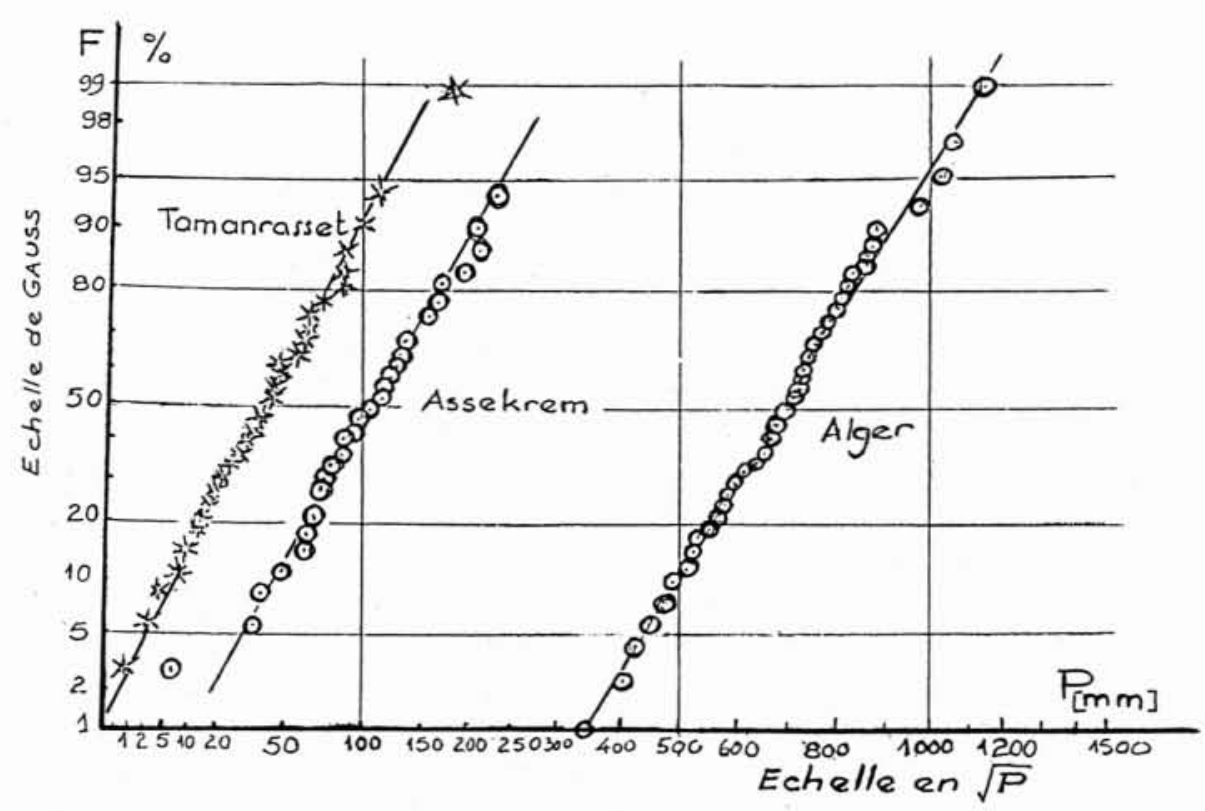

2. Fonction de répartition des pluies annuelles.
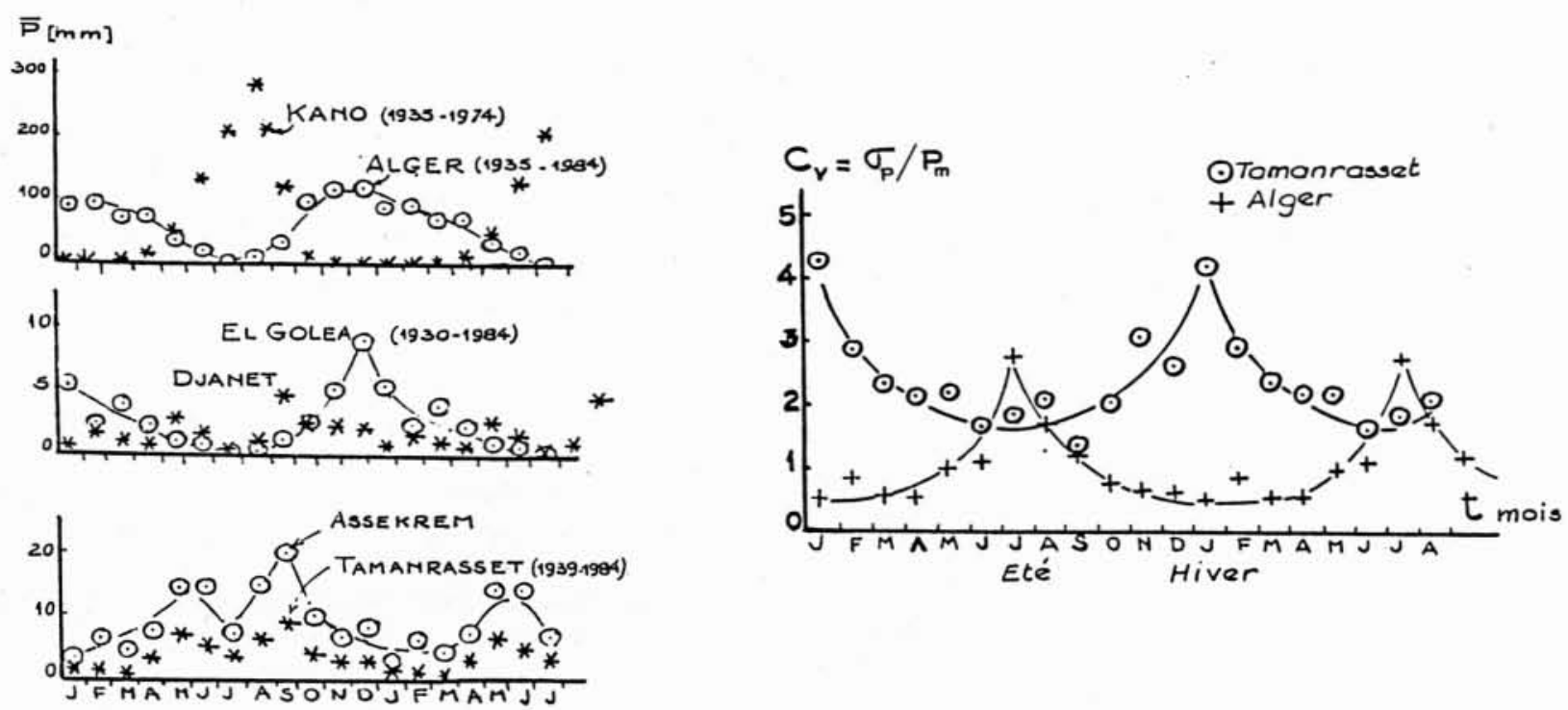

3. Pluies mensuelles moyennes.

4. Variabilité des pluies mensuelles. 


\section{Les critères de la sécheresse au Sahara}

\subsection{Les aléas de la pluviométrie annuelle}

La planche 2 montre les graphes des fonctions de répartition des pluies annuelles $P$ de quelques stations sahariennes en comparaison avec les résultats afférents à Alger.

Bien entendu les moyennes des pluies annuelles sont piteusement plus faibles sur le Sahara mais il y a plus : la forme des graphes est différente. L'allure gaussienne étayée par le théorème central limite qui est presque de règle pour les stations de zone tempérée est fortement remise en question par la dissymétrie de l'histogramme et même son aplatissement. Pour tenir compte de la spécificité de forme des graphes des stations sahariennes il a été proposé (MATARI, 1988) l'anamorphose $\sqrt{P}$ (habituelle par exemple pour les graphes intensitédurée-fréquence en hydraulique urbaine). Cette transformation assure une rectification convenable des graphes dans un diagramme gaussien (planche 2). A partir de ces graphes, on va pouvoir s'intéresser aux sécheresses dont la gravité sera caractérisée par la dispersion.

Cette dispersion peut être reliée à plusieurs critères par exemple le coefficient de variation rapport de l'écarttype sur la moyenne $s_{p} / \bar{P}$ ou le rapport à la moyenne de la différence de quantiles extrêmes. J. DUBIEF a choisi d'utiliser les quartiles. Mais si l'on reprend la classification de R. LAMBERT (1983) en années très humides, moyennes, sèches et très sèches, on utilisera la différence entre premier et dernier quintiles.

Utiliser un paramètre de dispersion pour caractériser les risques de sécheresse revient à admettre implicitement une certaine symétrie de la fonction de répartition. $\mathrm{Si}$ la dissymétrie est forte, alors il vaut mieux s'intéresser directement à la valeur d'un quantile, encore le premier quintile si on examine les sécheresses de période de retour de cinq ans dont parlait CHELlig (1974) ou le premier décile si on veut juger de conditions encore plus sévères pour les pâturages.

\subsection{L'hétérogénéité de la distribution de la pluie au cours d'une année}

La figure 3 déjà présentée donne une idée de l'évolution des moyennes mensuelles en fonction du site. A côté de l'aspect chamelier des stations du Hoggar, El Goléa par exemple présente en décembre une valeur maximale semblable à celle de Tamanrasset (plus de $8 \mathrm{~mm}$ ) mais placée entre de longues périodes de disette.

Cette figure 3 peut donner le change car en définitive la sécheresse habituelle d'été n'est guère plus sévère à El Goléa qu'à Alger. Mais d'une année à l'autre la pluie mensuelle d'un mois donné peut fortement varier. Témoin la figure 4 des coefficients de variation $C_{v}=$ $s_{m} / P_{m}$ qui fait apparaitre des valeurs énormes signe d'une dispersion détruisant toute prévision et d'hétérogénéité temporelle bien caractéristique des sécheresses désertiques.

A notre surprise, pour le phénomène si imprévisible et erratique de la pluie sur le Hoggar, le paramètre statistique que constitue le coefficient de variation men- suel suit un graphe d'une régularité déconcertante et la similitude entre les deux stations de Tamanrasset et de l'Assekrem est évidente en dépit de la forte différence d'altitude. Même constatation pour Alger et El Goléa dont les graphes présentent même forme que ceux de Tamanrasset et Assekrem mais sont déphasés d'environ une demi-année. Djanet avec ses deux bosses participe aux tendances des deux groupes. Sans prétendre reconstituer le mammouth climatologique à partir d'une dent orageuse, il semble cependant que le coefficient de variation présente une telle stabilité qu'elle en fait un bon paramètre statistique d'appréhension de l'hétérogénéité temporelle et donc des risques de sécheresses. Le coefficient de variation varie grosso modo en sens contraire de la moyenne mensuelle. Il y a plus d'aléa donc plus de risque de sécheresse les mois où il pleut moins en moyenne. Ceci avait déjà été bien constaté pour la distribution spatiale des pluies annuelles (DUBIEF, 1963, Matari, 1988). Un essai de corrélation $\left(C_{v}, P\right)$ fait certes apparaître la tendance mais assez floue au milieu d'un nuage effiloché.

Cette absence complète de pluie sur plusieurs mois est déjà vraiment un signe de sécheresse et nous en reparlerons plus loin avec la longueur des épisodes secs. Mais J. DUBIEF nuance encore en parlant de sécheresse efficace c'est-à-dire de longues périodes sans pluies suffisantes (ou efficaces) pour assurer la rénovation des pâturages. Pour cerner cette circonstance, on peut s'intéresser à la statistique des périodes où la pluie cumulée dépasse un certain seuil par exemple au graphe des mois participant à des triplets où au moins la pluie d'un mois dépasse $10 \mathrm{~mm}$ (DUBIEF, 1963) ou encore comme l'a proposé C.P. PEGUY utiliser la statistique des hauteurs de pluies mensuelles des pluies en 24 heures supérieures à un certain seuil $(5,10$ ou $15 \mathrm{~mm})$. En effet, en été la température élevée stimule une forte évaporation à laquelle n'échapperont pas les gouttes éparses d'une averse parcimonieuse. Chose surprenante, les résultats présentés sur ce critère par H. DUBIEF pour différentes stations sahariennes algériennes, font apparaître des ensembles non vides même pour le seuil de $20 \mathrm{~mm}$, ce qui prouve statistiquement que les fortes pluies ne sont pas inconnues au Sahara.

\subsection{La longueur des séquences sèches}

Le nombre de jours successifs sans pluie est évidemment un des critères quantitatifs le plus populaire de la sécheresse. Leur statistique (MATARI, 1988) fait apparâ̂tre des nombres de jours de pluie moyens annuels si faibles qu'on ne sera pas surpris de découvrir, compte tenu des énormes aléas du désert, des séquences sèches interminables.

Le coefficient de variation afférent au nombre de jours de pluie annuel n'est pas énorme $(0,47$ à Tamanrasset et 0,67 à Djanet station la plus sèche). Son inverse varie grosso modo de manière linéaire avec le nombre de jours de pluie :

$$
\frac{1}{C_{v}}=1+0,0095 n_{H} .
$$


Tableau 2. Longueurs moyennes en jour des séquences sèches et pluvieuses.

\begin{tabular}{|c|c|c|c|c|c|c|}
\hline \multirow{2}{*}{} & \multicolumn{2}{|c|}{ ANNEE } & \multicolumn{2}{c|}{ SAISON } & \multicolumn{2}{c|}{ SAISON } \\
\cline { 2 - 7 } & & & & & & \\
\hline TOULOUSE & 3,9 & 2,5 & 3,5 & 2,5 & 4,1 & 2,2 \\
\hline ALGER & 6,3 & 2,5 & 4,1 & 2,7 & 10,1 & 2,0 \\
\hline EL GOLEA & 42,0 & 1,3 & 26,1 & 1,3 & 101 & 1,2 \\
\hline TAMANRASSET & 33,5 & 1,5 & 19,2 & 1,5 & 113 & 1,7 \\
\hline ASSEKREM & 19,0 & 2,00 & 12,5 & 1,8 & 37 & 2,6 \\
\hline KANO & 8,6 & 1,6 & 3,6 & 1,6 & 16 & 1,5 \\
\hline
\end{tabular}

On peut se poser la question de savoir si les rares jours de pluie sont isolés ou rassemblés. La statistique des longueurs de séquences pluvieuses présentée dans le tableau 2 (MATARI, 1988) montre qu'il n'y a pas de différence sensible entre le semestre considéré comme le plus pluvieux et le reste de l'année.

Plus parlantes sur les sécheresses sont les longueurs de séquences sèches. Les records sont effrayants : 262 jours à l'Assekrem, 280 à El Goléa et 358 jours à Tamanrasset pour la période récente de 1955 à 1984. Au Hoggar les séquences maximales ont été observées en 1973 où on a recueilli seulement $0,9 \mathrm{~mm}$ à Tamanrasset et $8 \mathrm{~mm}$ sur le plateau de l'Assekrem à 2300 mètres.

En période dite sèche, la moyenne de la longueur de séquence sèche est presque de quatre mois à Tamanrasset et seulement d'un peu plus d'un mois à l'Assekrem ce qui indique clairement l'influence de l'altitude dans la région du Hoggar.

Un essai de modélisation de l'enchaînement des jours secs et des jours pluvieux a été tenté à l'aide des chaînes de Markov (MATARI, 1988) dont l'application est tout de même délicate vu la forte variabilité et il y a une nette divergence pour les séquences particulièrement longues (par exemple au-delà de 100 jours à Tamanrasset pour une seule distribution annuelle). Un ajustement à l'aide d'une loi binomiale tronquée (MARTIN, GalloY et LEBRETON, 1982) donne une meilleure proximité d'avec les graphes expérimentaux mais c'est bien normal puisque d'une part on dispose de plus de paramètres ou degrés de liberté et que, d'autre part, on contraint directement l'expression analytique à suivre les longueurs de séquence alors qu'avec les matrices de Markov, on fait un pari sur l'enchaînement des jours, hypothèse locale, dont on déduit le résultat global qu'est la fonction de distribution des longueurs de séquence.

Comparé aux résultats obtenus pour le Sud-Ouest de la France (ARnAud/THIRriot, 1985), le coefficient de corrélation binaire d'un jour sur l'autre n'est tout de même pas ridiculement faible puisqu'on obtient pour les coefficients annuels : $r=0,30$ à Tamanrasset, $r=0,43$ à l'Assekrem mais seulement $r=0,20$ à El Goléa pour $r=0,36$ à Toulouse et $r=0,44$ à Alger.

\section{Conclusion}

Alors peut-on parler de sécheresse au Sahara? Bien sûr ! la tradition séculaire des nomades l'affirme et les statistiques le confirment. Dans ce climat fantasque, les observations scientifiques et les mesures détruisent peu à peu le mythe d'un comportement météorologique indicible. Bien sûr, des lourds nuages qui traînent sans précipitation, une luminosité brûlante après des ouragans qui lessivent sol et maisons, la rareté et la pauvreté des pluies, un réseau hydrographique vu d'avion puissant mais sans eau ont longtemps fait croire que le Sahara météorologique était hors des normes et hors toute classification. Mais J. DUBIEF avec l'humour et la sérénité d'un homme du désert conclut que cette réputation du Sahara soumis aux règles météorologiques était surfaite. Il y a des saisons au Sahara mais leur localisation calendaire change avec la situation géographique avec un hiver humide au Nord et $\mathrm{sec}$ au Sud. Les aléas sont plus violents qu'ailleurs parce qu'ils sont exacerbés par l'aridité et dans le désert comme sur le pourtour méditerranéen le coefficient de variation varie en raison inverse de la hauteur de pluie annuelle moyenne.

Les longues périodes sèches fléaux des pâturages et des troupeaux qui alors rabotent encore plus le sol ingrat et les pousses squelettiques peuvent aussi être fichées et ajustées. La sécheresse du Sahara aujourd'hui peut se lover dans l'ordinateur, les intervalles de confiance des fonctions de répartition se préciseront peu à peu avec l'accumulation des observations de qualité. Mais le pays est immense et si divers dans sa nudité de l'Atlantique à l'océan Indien, qu'il fera rêver encore longtemps le météorologue.

Tout savoir sur la statistique des sécheresses ne permet pas de les guérir et leur insécurité lancinante jointe aux efforts politiques de sédentarisation vont réduire l'épopée des pasteurs nomades à la nostalgie des méharées d'antan. Restera encore la projection vers la liberté dangereuse des grands espaces arides, pour le citadin emprisonné dans le confort bruyant, solitaire et pollué des mégalopoles qui perdent leur âme. Vers ce Sahara dont la fascination a forgé tellement d'hommes exceptionnels tels de Foucauld, Theodore Monod, Jean Dubief pour qui l'observation scientifique confinait à la méditation. 


\section{Remerciements}

Tout au long de ce rapport, le lecteur aura vu tout ce que les auteurs doivent à Jean DUBIEF, à son œuvre, à sa personnalité et à son aide amicale puisque aujourd'hui encore, à quatre-vingt-six ans, il a su apporter suggestions et documentations originales. Avec Théodore MONOD et bien d'autres certainement, il est la preuve que le désert est une ascèse du bien vieillir.

Tout aussi efficace a été l'aide apportée par Monsieur
TABET, directeur enthousiaste de l'Institut hydrométéorologique d'Oran qui par amitié a utilisé son temps précieux à explorer ses archives personnelles pour nous faire profiter d'une documentation difficile à trouver par ailleurs.

Enfin nous voulons remercier l'Institut méditerranéen de l'eau et tout particulièrement son délégué général Monsieur Louis POTIE, qui ont été un catalyseur de la petite équipe "Crues et Sécheresses » de l'Institut de mécanique des fluides de Toulouse.

\section{Bibliographie}

ARNAUD M. (1985) : Contribution à l'étude stochastique markovienne des précipitations dans le bassin Adour-Garonne. Thèse présentée à l'Institut National Polytechnique de Toulouse.

Chellig R. (1974): Communication sur les pâturages steppiques. Séminaire international sur le pastoralisme. Alger 22-28 avril.

Claudin J. (1974) : Carte d'aptitude du milieu pour la mise en valeur pastorale. Séminaire international sur le pastoralisme. Alger 22-28 avril.

Dubief J. (1963): Le climat du Sahara. Tomes 1 et II. Université d'Alger. Institut de Recherches Sahariennes. Publić avec le concours du CNRS (Paris).

EAgleson P. (1970): Dynamic hydrology. Mac Graw-Hill. Book Company. New York Londres.

Eydoux H.P. (1943): L'homme et le Sahara. Collection Géographic Humaine. Gallimard NRF. 207 pages.

Gautier E. F. (1928): Le Sahara. Payot. Paris. 232 pages.

HADDARI R. (1974) : Nomadisme et achaba dans la wilaya des oasis. Séminaire international sur le pastoralisme. Alger 22-28 avril.

KERBAA F. (1974): Etude de quelques facteurs d'intensification des producteurs fourragers en milieu steppique. Séminaire international sur le pastoralisme. Alger 22-28 avril.

LAMBERT R. (1983): Géographie du cycle de l'eau. Université de Toulouse II Le Mirail. Service d'enseignement à distance.

Martin, Galloye, Lebreton A. (1982) : Analyse des séquences de jours secs consécutifs. Application à 31 postes du réseau climatologique français. La Météorologie $\mathrm{VI}^{\mathrm{c}}$ séric nं 28 , pp. 5-28.

MATARI A. (1988): Essai de représentation markovienne de la pluviométrie au Sahara. Thèse de Diplôme de Recherche Universitaire. Institut National Polytechnique de Toulouse. 27 octobre.

ThIRriot C., ARNAud M. (1985): Leçon et ambiguité d'une longue séric pluviométrique à Toulouse. Journal Rech. Atmosph. $\mathrm{n}^{\circ} 1$, pp. 25-45.

THIRRIOT C. (1987) : Sur l'information apportée par l'accroissement de l'ordre des chaînes de Markov discrètes utilisées pour la représentation de l'état pluviométrique journalier. CRAS Paris, t. 305. Séric II, pp. 1081-1084.

THIRRIOT C. (1967): Normales climatologiques relatives aux stations climat et climat ship pour la période 1931-1960. OMN Genève. 